\title{
Treatment outcomes and prognostic factors of cervical cancer at South Egypt Cancer Institute
}

\begin{abstract}
Background: The aim of this retrospective study was to analyse the clinicopathological factors of cervical cancer and its prognostic significance, various treatment adopted and their outcome in our local sitting.

Methods: From January 2005 to December 2013, a total of 83 patient records were analyzed. As initial treatment, 16 patients underwent surgical resection and 61 patients received definitive radiotherapy, the remaining 6 patients had stage IV disease and received palliative treatment. Among 61 patients received definitive radiotherapy, forty six patients received chemotherapy concurrently with radiotherapy and external beam boost was given to 43 patients while brachytherapy boost was given to 18 patients. Thirteen patients had residual disease after definitive radiotherapy, nine of whom underwent salvage surgery and the remaining 4 patients were inoperable and received palliative treatment. A total of 16 operated patients received radiotherapy due to presence of risk factors.
\end{abstract}

Results: After median follow up period of 54 months (range $=8-108$ months), treatment failed in 37 patients $(48.0 \%)$. The pattern of failure included isolated local recurrence in 21 patients (27.3\%), isolated distant metastasis in 13 patients $(16.9 \%)$ and mixed in 3 patients (3.9\%). The 5-year overall survival (OS) and disease free survival (DFS) were $53.7 \%$ and $46.3 \%$ respectively. In univariate analysis, tumor size $(\mathrm{p}=.033)$, stage $(\mathrm{p}=.001)$ and type of initial treatment $(\mathrm{p}=.034)$ were significant prognostic factors for OS while clinical stage was the only statistically significant prognostic factor for DFS ( $\mathrm{p}=.009)$. In multivariate analysis, advanced stage was independent prognostic factor for poor OS $(\mathrm{p}=.001)$ and DFS $(\mathrm{p}=.003)$.

Conclusion: Most of cervical cancer patients at our Institute were seen at late stage which reflects lack of effective cervical screening program and necessitating the need for organized referral system to regional center to ensure optimal combined treatment.

Keywords: cervical cancer, chemoradiation, prognostic factors, radical hysterectomy
Volume 2 Issue 6 - 2015

\author{
Mohamed Abouelmagd Salem, ${ }^{2}$ Alia M Attia, I \\ Gamal Amira ${ }^{3}$ \\ 'Radiation Oncology Department, South Egypt Cancer Institute, \\ Assiut University, Egypt \\ ${ }^{2}$ Surgical Oncology Department, South Egypt Cancer Institute, \\ Assiut University, Egypt \\ ${ }^{3}$ Surgical Oncology Department, National Cancer Institute, \\ Cairo University, Egypt
}

Correspondence: Alia M Attia, Radiation Oncology Department, South Egypt Cancer Institute, Al-Mthaq St., Assiut 7I I I I, Egypt, Tel 20-1000004857, Fax 20-882348609, Tel 201018116193, Fax 20-882348609,

Email aliamohamadattia@yahoo.com

Received: June 12, 2015 | Published: June 22, 2015

\section{Introduction}

Cervical cancer is one of the common gynecological malignancies, with nearly 500,000 newly diagnosed cases each year of which more than $90 \%$ in developing countries. ${ }^{1}$ The highest incidence of cervical cancer occurs in Central and South America, Eastern Africa, South and South-East Asia, and Melanesia. ${ }^{2}$ Invasive cervical cancer is the most common cause of cancer deaths in Africa accounting for $10.4 \%$, which represents one in five of all cancer deaths in African women. ${ }^{3}$

The main reasons for higher incidence and mortality of the cervical cancer in developing countries include lack of awareness of cervical cancer in the general population and health care providers and lack of effective screening program to detect and treat early stage of the disease. ${ }^{4}$ There are many clinicopathological factors affecting recurrence in cervical cancer which become the main reason for decreased survival rate and these include, clinical stage, histological grade, and treatment adopted. .,6 $^{5}$

In developing countries, few studies are available describing the incidence of cervical cancer, disease stage, management and treatment outcome of these patients. ${ }^{7,8}$ Therefore the aim of this study is to establish the database of patients with cervical cancer taking into account the clinicopathological pattern of cervical cancer, highlight different treatment modalities adopted in the management of this disease in our local setting and its effect on disease free survival and overall survival, and evaluate clinicopathological risk factors affecting treatment outcome and survival.

\section{Patients and methods}

This retrospective hospital based study included 83 patients diagnosed with cervical cancer and presented to South Egypt Cancer Institute (SECI) from January 2005 to December 2013. The clinical records of these patients were retrospectively analyzed and the following variables were recorded: age, sign and symptoms, parity, histopathology including squamous cell carcinoma or adenocarcinoma, stage based on International Federation of Gynaecology and Obstetrics classification ${ }^{9}$ (IA-IVB), grade (well, moderate, poor), primary treatment plan (Surgery, radiotherapy or both), the date of diagnosis and last follow up visit and treatment outcomes (date and site of relapse, date of death). Out of 83 patients, 6 patients presented with stage IVB cervical cancer and they were excluded from survival analysis.

\section{Treatment}

Therapeutic strategies were selected for individual patients. In stage I-IVA patients, various treatments such as the combination of surgery and radiotherapy, and chemoradiation therapy were used. Stage IVB patients, various treatment combinations of chemoradiation, palliative radiotherapy, palliative chemotherapy and best supportive care were used. 
For surgery, radical hysterectomy with pelvic lymphadenectomy was performed in some patients while pelvic excentration was performed in others. Radiotherapy was given to patients either definitively or postoperatively; patients with deep muscular involvement, vascular invasion, tumor diameter larger than $4 \mathrm{~cm}$, positive lymph node or positive surgical margin were treated with postoperative radiotherapy with or without concurrent chemotherapy. Patients received definitive radiotherapy, whole pelvic irradiation using external beam radiotherapy with $10-15 \mathrm{MV}$ photon of 1.8 Gy per fraction, 5 times per week for a total dose of 50.4 Gy. After external beam radiotherapy, patients were assessed for possibility of intracavitary application. If possible, patients received intracavitary brachytherapy to a total dose of 30Gy / 6 Gy per fraction. Patients with parametrial infiltration, received parametrial boost of 10 Gray in 5 fractions with central blocking. If intracavitary brachytherapy was not possible, additional dose of external beam radiotherapy was given to a total dose of $60 \mathrm{~Gy}$. Postoperative radiotherapy was delivered to a total dose of $50.4 \mathrm{~Gy} / 5$ fractions per week. During concurrent chemoradiation blood transfusion were allowed to keep hemoglobin level $\geq 10 \mathrm{~g} / \mathrm{dl}$. Chemotherapy dose was reduced to $30 \mathrm{mg} / \mathrm{m}^{2}$ when patients developed grade 1 or 2 hematological toxicity and it was held in case of grade 3 or 4 hematological toxicity.

\section{Follow up}

After treatment, all patients were followed up regularly every 3 months in the first 2 years and every 6 months thereafter by history and pelvic bimanual examinations to assess response to treatment. Chest x-ray, computerized tomography (CT) scan of the pelvis and abdomen of the pelvis were done every 6 months in the first year and yearly thereafter. Persistent or recurrent disease was documented by imaging studies or biopsy if possible.

Treatment related toxicity was assessed weekly during treatment and at the time of each visit using National Cancer Institute Common Terminology Criteria for Adverse Effect v 3.0. ${ }^{10}$

\section{Statistical analysis}

DFS was measured from the date of diagnosis to the date of local recurrence, or distant metastasis or last follow up. OS was measured from the date of diagnosis to the date of death from any cause or to the date of the last follow-up. DFS and OS curves were estimated using the Kaplan-Meier method. ${ }^{11}$ Survival data between groups were compared with the Log rank test for univariate analysis and Cox regression analysis for multivariate analysis. The $95 \%$ confidence interval (CI) was calculated for the risk ratios for each of the significant prognosticators. All $\mathrm{P}$ values $<0.05$ considered to be significant. All analyses performed using the Statistical Package for Social Sciences software, SPSS, version 18.0 (SPSS inc. Chicago, Illinois, USA).

\section{Ethical considerations}

Data were stored securely and made available only to persons conducting the study. All patients participated in this study were coded in numbers instead of names. Treatment decision was approved by institutional review board at our center.

\section{Results}

The characteristics of 83 patients with cervical cancer were listed in Table 1. The age of the patients ranged from 28-75 years with a median age of 55 years. Vaginal bleeding (Postcoital, intermenstrual, or postmenopausal) was the most common presenting symptom which was reported by 63 patients ( $75.9 \%)$. Squamous cell carcinoma was the most common histopathological tumor and it was encountered in 77 patients $(92.8 \%)$. According to the FIGO classification, the majority of the patients $(42.2 \%)$ presented with stage III disease.

\section{Treatment}

\section{Surgery}

Out of 77 patients (stage IB-IVA), 16 (20.8\%) patients underwent primary surgical resection. Radical hysterectomy and pelvic lymphadenectomy was performed in 15 patients and only one case $(1.3 \%)$ underwent posterior pelvic excentration.

\section{Combined modality}

Sixty one patients (79.2\%) out of 77 patients (stage I-IVA) received external beam radiotherapy as an initial phase of treatment. External boost radiotherapy was given to 43 patients $(70.5 \%)$ and brachytherapy boost to 18 patients $(29.5 \%)$. Concurrent chemotherapy was given to 46 patients $(75.4 \%)$. The remaining 15 patients $(24.6 \%)$ were ineligible to receive chemotherapy concurrently with radiotherapy due to renal impairment and treatment related toxicity.

Out of 61 cases received definitive radiotherapy, 13 patients $(21.3 \%)$ had residual or progressive disease after the whole course of radiotherapy (11 patients received external beam radiotherapy as a boost and 2 patients received brachytherapy as a boost). Nine out of 13 patients, underwent salvage radical hysterectomy and pelvic lymphadenectomy, while the remaining 4 patients were inoperable and received palliative chemotherapy and they died. Four out of 6 stage IVB patients received palliative chemotherapy and or palliative radiotherapy. The remaining 2 cases presented with para aortic lymph node as the only site of metastasis and received definitive chemoradiotherapy.

\section{Adjuvant therapy}

A total of 16 operated patients $(20.8 \%)$ received whole pelvic external radiation within 1 month of surgical resection and 3 of them received neoadjuvant chemotherapy. Adjuvant chemoradiotherapy was given as a result of positive surgical margins in 4 patients $(25 \%)$, positive pelvic lymph nodes in 4 patients $(25.0 \%)$ and parametrial invasion in 5 patients $(31.2 \%)$. Adjuvant radiotherapy was given in tumor diameter larger than $4 \mathrm{~cm}$ in 7 patients $(43.7 \%)$, deep stromal invasion in 5 patients $(31.2 \%)$ and lymphovascular space invasion in 2 patients (12.5\%) (Table 1).

\section{Pattern of failure after treatment and follow up}

After median follow up period of 54 months (range $=8-108$ months), treatment failed in 37 patients $(48.0 \%)$ out of 77 patients with stage IB through IVA cervical cancer. The patterns of treatment failure were, isolated local recurrence in 21 patients $(27.3 \%)$, isolated distant metastasis in 13 patients $(16.9 \%)$ and local recurrence with distant metastasis in 3 patients (3.9\%) (Table 2).

Among 21 patients who developed isolated local recurrence, 12 patients were candidate for surgical resection. Pelvic excentration was performed in 9 patients while radical hysterectomy was performed in 3 patients. The remaining 9 cases with isolated local recurrence were inoperable and received palliative chemotherapy. Five out of 12 patients with surgically removed isolated local recurrence were alive and free of the disease, and the remaining 7 patients died from surgical complications. All 9 inoperable cases had progressive disease with poor general conditions that required best supportive care and they died. 
Table I Characteristics of 83 patients with cervical cancer

\begin{tabular}{|c|c|c|}
\hline Variable & Number & Percentage \\
\hline Age & 55 & 45.8 \\
\hline Median & $28-75$ & 54.2 \\
\hline Range & 38 & \\
\hline$<50$ & 45 & \\
\hline \multicolumn{3}{|l|}{$\geq 50$} \\
\hline Duration of Symptoms & 8 & \\
\hline Mean & $2-48$ & \\
\hline \multicolumn{3}{|l|}{ Range } \\
\hline Pretreatment Hemoglobin Level & 34 & 41 \\
\hline$<10 \mathrm{~g} / \mathrm{dl}$ & 49 & 59 \\
\hline \multicolumn{3}{|l|}{$\geq 10 \mathrm{~g} / \mathrm{dl}$} \\
\hline Symptoms & 38 & 45.8 \\
\hline Vaginal Bleeding & 15 & 18.1 \\
\hline Vaginal Discharge & 9 & 10.8 \\
\hline Vaginal Bleeding \& Urinary Symptoms & 6 & 7.2 \\
\hline Urinary symptoms & 16 & 19.3 \\
\hline \multicolumn{3}{|l|}{ Pain \& vaginal bleeding } \\
\hline Histology & 77 & 92.8 \\
\hline Squamous cell carcinoma & 6 & 7.2 \\
\hline \multicolumn{3}{|l|}{ Adenocarcinoma } \\
\hline Tumor Size & 21 & 25.3 \\
\hline$\leq 4 \mathrm{~cm}$ & 62 & 74.7 \\
\hline \multicolumn{3}{|l|}{$>4 \mathrm{~cm}$} \\
\hline Lymph Node Metastasis & 53 & 63.9 \\
\hline Negative & 30 & 36.1 \\
\hline Positive & 1 & 1.2 \\
\hline FIGO Stage & 9 & 10.8 \\
\hline$|B|$ & 6 & 7.2 \\
\hline IB2 & 20 & 24.1 \\
\hline IIA & 7 & 8.4 \\
\hline IIB & 28 & 33.7 \\
\hline IIIA & 6 & 7.2 \\
\hline IIIB & 6 & 7.2 \\
\hline \multicolumn{3}{|l|}{ IVA } \\
\hline \multicolumn{3}{|l|}{ IVB } \\
\hline Grade & 9 & 10.8 \\
\hline Well Differentiated & 34 & 41 \\
\hline Moderately Differentiated & 40 & 48.2 \\
\hline \multicolumn{3}{|l|}{ Poorly Differentiated } \\
\hline Treatment Modality Adopted & 16 & 19.3 \\
\hline \multicolumn{3}{|l|}{ Surgery: } \\
\hline \multicolumn{3}{|c|}{ a. Radical Hysterectomy and Pelvic Lymphadenectomy (I5 patients) } \\
\hline b. Pelvic Excentration (I patient) & 63 & 75.9 \\
\hline $\begin{array}{l}\text { c. Neoadjuvant Chemotherapy ( } 3 \\
\text { patients) }\end{array}$ & 4 & 4.8 \\
\hline \multicolumn{3}{|c|}{ d.Adjuvant Chemoradiotherapy (I6 patients) } \\
\hline \multicolumn{3}{|l|}{ Definitive Chemoradiotherapy } \\
\hline Palliative Radiotherapy \&/or Chemoth & & \\
\hline
\end{tabular}

Table 2 Pattern of treatment failure of 77 patients (stages IBI-IVA) with cervical cancer

\begin{tabular}{lll}
\hline Variables & Number & Percentage \\
\hline Total Recurrences & 37 & 48 \\
Isolated LR & 21 & 27.3 \\
Total DM & 16 & 20.8 \\
Isolated DM & 13 & 16.9 \\
DM + LR & 3 & 3.9 \\
Site of DM & & \\
Paraortic Lymph Nodes & 3 & \\
Liver & 4 & \\
Lung & 3 & \\
Liver \& Paraortic Lymph Nodes & 3 \\
Lung \& Paraortic lymph Nodes & 1 \\
Liver \& Bone & 1 \\
Lung \& Bone & 1 & \\
\hline
\end{tabular}

Abbreviations: EBRT, external beam radiotherapy; $\mathrm{CT}$, chemotherapy; Brachy, brachytherapy

The number of distant metastasis was one in 10 patients while 6 patients had 2 sites of distant metastases. The sites of distant metastases included para aortic lymph nodes in 7 patients $(9.1 \%)$, liver metastases in 8 patients $(10.4 \%)$, lung metastasis in 5 patients $(6.5 \%)$, and bone metastasis in 2 patients $(2.6 \%)$. Patients who developed isolated distant metastasis and local recurrence with distant metastasis received palliative radiotherapy and or chemotherapy. All of metastatic cases died of their disease.

\section{Survival}

The 5-year OS and DFS of the 77 patients (excluding stage IVB patients) with cervical cancer were $53.7 \%$ (95\% CI $62.3 \%$ to $79.9 \%)$ and $46.3 \%$ (95\% CI $50.7 \%$ to $70.8 \%$ ) respectively (Figur ${ }^{1}$ es $1 \& 2$ ). There were no treatment related deaths.

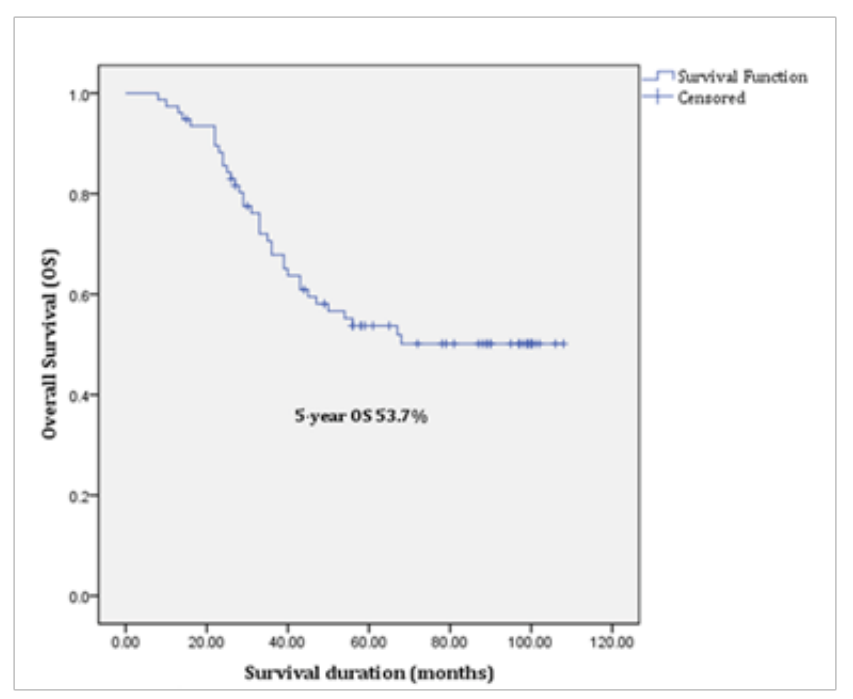

Figure I 5-year overall survival of 77 patients with cervical cancer. 


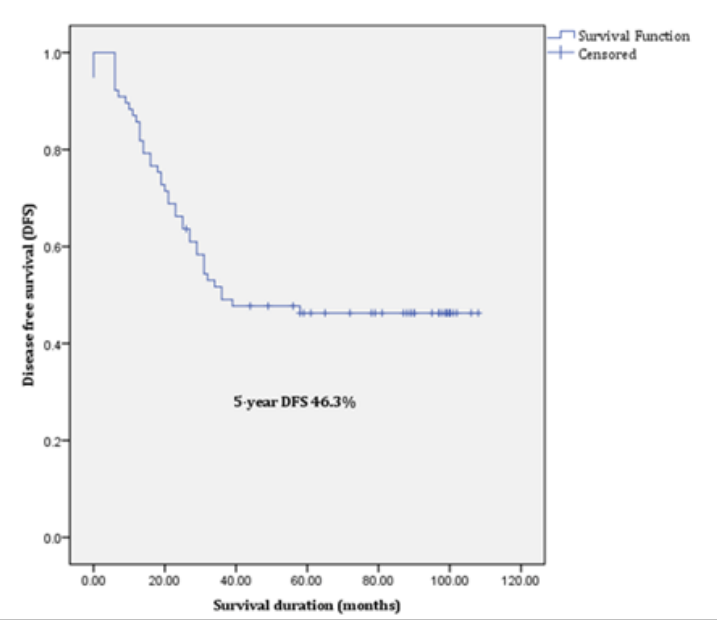

Figure 25 -year disease free survival of 77 patients with cervical cancer.

\section{Prognostic factors for survival}

Prognostic factors, such as age, pretreatment hemoglobin level, histology, tumor size, lymph node involvement, FIGO stage, grade and type of initial treatment adopted were analyzed. On univariate analysis, tumor larger than $4 \mathrm{~cm}(\mathrm{P}=0.033)$, advanced stage ( $\mathrm{p}=$ $0.001)$ and surgical resection as an initial treatment $(\mathrm{p}=0.034)$ showed statistically significant decrease in OS. Patients with advanced stage $(\mathrm{p}=0.009)$ had statistically significant poor DFS on univariate analysis (Table 3). Multivariate analysis showed that advanced stage was the only independent prognostic factor for poor OS and DFS; (HR= 3.237; $95 \% \mathrm{CI}=1.609-6.513 ; \mathrm{p}=.001)$ and $(\mathrm{HR}=2.694 ; 95 \% \mathrm{CI}=$ $1.406-5.161 ; p=0.003)$ respectively. Figure $3 \& 4$ showed KaplanMeier analysis of overall survival and disease free survival according to stage.

Table 3 Univariate analysis for DFS and OS in 77 patients with cervical cancer

\begin{tabular}{lll}
\hline Variables & \multicolumn{2}{l}{ 5-Year Survival Rates (\%) } \\
\hline & DFS & OS \\
Age & 40.7 & 48.9 \\
$<55(n=36)$ & 51.2 & 58 \\
$\geq 55(n=4 I)$ & $P=0.37$ & $P=0.22$ \\
Pretreatment Hemoglobin Level & 39.1 & 48.6 \\
$<$ I0 $(n=32)$ & 51.1 & 56.9 \\
$\geq$ I0 $(n=45)$ & $P=0.44$ & $P=0.49$ \\
Histology & 48.8 & 57.1 \\
Squamous Cell Carcinoma $(n=71)$ & 16.7 & 16.7 \\
Adenocarcinoma ( $n=6)$ & $P=0.12$ & $P=0.09$ \\
Tumor Size & 57.1 & 73.5 \\
$\leq 4 c m(n=2 I)$ & 42.1 & 47.1 \\
$>4$ cm ( $n=56)$ & $P=0.28$ & $P=0.033$ \\
Lymph Node Metastasis & 45.4 & 56.9 \\
Negative $(n=52)$ & 48 & 47.7 \\
Positive $(n=25)$ & $P=0.96$ & $P=0.57$ \\
FIGO stage & 90 & 88.9 \\
I $(n=10)$ & 50 & 61.7 \\
II $(n=26)$ & 36.7 & 45.5 \\
\hline
\end{tabular}

Table continued..

\begin{tabular}{lll}
\hline Variables & \multicolumn{2}{l}{ 5-Year Survival Rates (\%) } \\
\hline III $(n=35)$ & 16.7 & 16.7 \\
IV $(n=6)$ & $P=0.009$ & $P=0.00 I$ \\
Grade & 33.3 & 44.4 \\
Well Differentiated $(n=9)$ & 49 & 62.4 \\
Moderately Differentiated $(n=32)$ & 47.2 & 49 \\
Poorly Differentiated $(n=36)$ & $P=0.79$ & $P=0.39$ \\
Initial Treatment Adopted & 68.2 & 71.8 \\
Surgery $(n=16)$ & 27.3 & 30.3 \\
-EBRT alone $(n=I 1)$ & 34.1 & 38.4 \\
-CT+EBRT $(n=32)$ & 50 & 50 \\
-EBRT + Brachy $(n=4)$ & 64.3 & 69.3 \\
-CT+EBRT+ Brachy $(n=14)$ & $P=0.07$ & $P=0.034$ \\
\hline
\end{tabular}

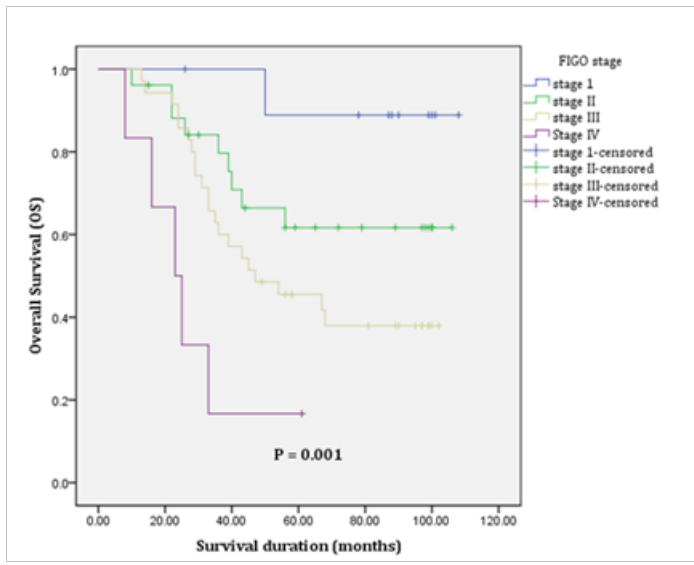

Figure 3 Kalpan- Meier analysis of overall survival per stage.

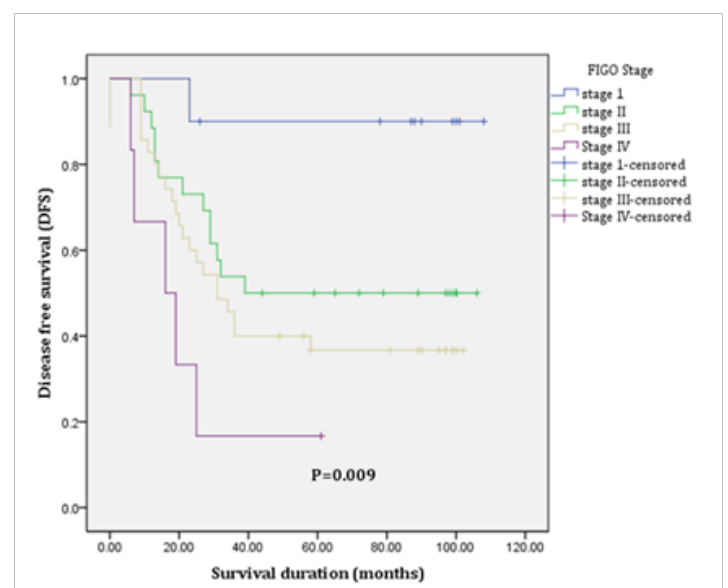

Figure 4 Kalpan-Meier analysis of disease free survival per stage.

\section{Complications of surgery}

Immediate postoperative complications among 24 patients $(16$ patients underwent surgery as an initial treatment and the remaining 9 patients underwent salvage surgery) included, urinary tract infection in 3 patients $(12.5 \%)$ and wound infection in 4 patients $(16.7 \%)$, small bowel obstruction in 2 patients $(8.3 \%)$. Three patients $(12.5 \%)$ developed fistula that required surgical intervention (one patient developed uretro-vaginal fistula and vesico-vaginal fistula occurred in 2 patients) all of whom underwent salvage radical hysterectomy and pelvic lymphadenectomy. 


\section{Treatment toxicity}

Acute toxicity was assessed weekly during radiotherapy and the most frequent acute low grade toxicities among 61 patients received definitive chemoradiotherapy were hematological in 28 patients $(45.9 \%)$, gastrointestinal in 25 patients $(41.0 \%)$, nausea and vomiting in 22 patients $(36.1 \%)$, skin in 15 patients $(24.6 \%)$ and genitourinary in 6 patients $(9.8 \%)$. Eleven patients (18.0\%) developed $\geq$ grade 3 hematological toxicity and 5 patients $(8.2 \%)$ had $\geq$ grade 3 gastrointestinal toxicity. As regard late toxicity (occurred after 90 days of the first day of radiotherapy), four patients (6.5\%) developed $\geq$ grade 3 gastrointestinal toxicity and 8 patients (13.1\%) developed $\geq$ grade 3 genitourinary toxicity.

\section{Discussion}

Majority of the patients (58\%) in our study were above 40 years old. Data from cancer registries in developing countries reported the same finding; $80 \%-90 \%$ of women with cervical cancer in the developing countries were above the age of $40 .{ }^{12}$ Eighty percent of women with cervical cancer in developing countries are diagnosed at advanced stages. ${ }^{13}$ In our study, 47 patients $(56.6 \%)$ presented with cervical cancer at stage III and IV, and these results were similar to other reports. ${ }^{14,15}$ This might be attributed to the lack of screening services in our country and women tend to seek medical advice only when they have developed symptoms. In England, 23.8\% of 382 women were diagnosed in stages III and IV ${ }^{16}$ which contributes to the longer survival. ${ }^{17}$

Many series reported pelvic failure rate of (20\%-25\%) and distant failure rate of $(10 \%-25 \%)$ after concurrent chemoradiotherapy. ${ }^{18-22}$ Our study reported local recurrence rate of $31.2 \%$, and distant metastasis of $20.8 \%$. The high local recurrence rate compared to the figures of the published series might be attributed to the high percentage of advanced stage in our patients $(56.6 \%)$. Another reason might be due to $15(24.6 \%)$ patients did not receive chemotherapy concurrently with radiotherapy as they were ineligible leading to poor outcome as a result of suboptimal treatment.

According to the American Cancer Society, the 5-year overall survival in the US is $72 \% .{ }^{17}$ The low 5 -year OS rates (53.7\%) found in our study was due to high number of cases presented with advanced disease, and treatment interruption of combined modalities which is essential for management of locally advanced disease. The reasons for treatment interruption was low hemoglobin level that required blood transfusion in order to continue chemoradiation treatment, patients received radiotherapy alone as they were ineligible for chemotherapy administration, patients travel to Cairo to receive brachytherapy course and due to crowdedness of the institution, waiting time was required for the start of brachytherapy. All these factors might contributed to high pelvic failure rate and consequently poor 5-year OS.

Radiotherapy or radical hysterectomy with bilateral pelvic lymphadenectomy for early stage cervical cancer yield comparable cure rate and survival. ${ }^{23-25}$ Landoni et al., ${ }^{26}$ reported 5-year OS in stage IB-IIA of $83 \%$ and Ayhan \& Tuncer ${ }^{27}$ reported 5 -year OS in stage IA, IB, IIA, and IIB of $(100 \%, 87.9 \%, 71 \%$, and $64 \%)$ respectively. ${ }^{27}$ Two series reported that, the 5-year OS rates in stage IIIB ranged from 25$48 \%{ }^{28}$ A survival analysis of women with cervical cancer in Brazil reported 5-year survival rates of $85 \%$ for stage I, $64.3 \%$ for stage II, $48.6 \%$ for stage III, and only $14 \%$ for stage IV..$^{29}$ In our study, 5-year OS for stage IB was $87.5 \%$; all of whom underwent surgical resection except one. The 5-year OS in stage IIA, IIB, IIIA, IIIB, and IVA was $(80 \%, 63.3 \%, 50.0 \%, 38.1 \%$, and $20 \%$ respectively).
There are several prognostic factors for cervical cancer that had been reported by several studies, such as clinical stage, tumor histology, size of primary tumor, depth of invasion, tumor grade, parametrium involvement, lymph node involvement, and lymphvascular space invasion (LVSI). ${ }^{30-35}$ In our study, univariate analysis revealed that tumor $\geq 4 \mathrm{~cm}(\mathrm{p}=0.033)$, advanced stage $(\mathrm{p}=0.001)$ and patients received definitive radiotherapy alone $(p=0.034)$ had worse OS, while advanced clinical stage was the only statistically significant prognostic factor for DFS (0.009). Multivariate analysis showed that advanced stage was the only independent prognostic factor for poor OS and DFS; $(\mathrm{HR}=3.237 ; 95 \% \mathrm{CI}=1.609-6.513 ; \mathrm{p}=.001)$ and $(\mathrm{HR}=2.694 ; 95 \% \mathrm{CI}=1.406-5.161 ; \mathrm{p}=0.003)$ respectively. Other risk factors were not significantly associated with poor survival; the reason might be attributed to the small number of patients enrolled in the study, which limit the power to detect any small effect of the treatment modalities adopted on the outcome.

Regarding the effect of initial treatment modalities adopted and survival, surgery and postoperative chemoradiotherapy resulted in statistically significant best 5 -year OS $(p=0.034)$. This is probably might be due to the fact that most patients underwent radical hysterectomy and bilateral pelvic lymphadenectomy had early stage of the disease compared to patients received chemoradiotherapy or radiotherapy alone.

\section{Conclusion}

Most of cervical cancer patients were seen at an advanced disease stage which leads to poor prognosis and outcome. After analyzing the age, pretreatment hemoglobin level, histology, tumor size, lymph node metastasis, FIGO stage, histological grades and initial treatment adopted of the 77 patients with cervical cancer using Cox regression model, we found that clinical stage was independent prognostic factors for poor OS and DFS. The preventable and curable nature of the disease necessitate the establishment of an effective screening program, education of women on the benefit of early diagnosis of the disease and consequently better treatment outcome. Establishment of an effective referral system from our Institute to regional center in Cairo, and increase the facilities and services provided for the patients care and management to alleviate the crowdedness and overloading at single referral hospital which in turn reduce the waiting time between the initial phase of external beam radiotherapy and brachytherapy.

\section{Acknowledgments}

None.

\section{Author contributions}

AM. Attia: Interpreting the data, and writing and critical revision of the article.

MA. Salem: Design and planning the research article.

G Amira: Critical revision of the article.

\section{Conflicts of interest}

The authors have no financial conflicts of interest to declare.

\section{Funding}

None.

\section{References}

1. Waggoner SE. Cervical cancer. Lancet. 2003;361(9376):2217-2225. 
2. WHO. Comprehensive cervical cancer control: a guide to essential practice. $2^{\text {nd }}$ ed. Geneva, Switzerland; 2006.

3. Munoz N, Bosch FX, de Sanjose S, et al. Epidemiologic classification of human papillomavirus types associated with cervical cancer. $N$ Engl $J$ Med. 2003;348(6):518-527.

4. Sankaranarayanan R, Budukh AM, Rajkumar R. Effective screening programmes for cervical cancer in low- and middle-income developing countries. Bull World Health Organ. 2001;79(10):954-962.

5. Gadducci A, Teti G, Barsotti C, et al. Clinicopathological variables predictive of clinical outcome in patients with FIGO stage Ib2-IIb cervical cancer treated with cisplatin-based neoadjuvant chemotherapy followed by radical hysterectomy. Anticancer Res. 2010;30(1):201-208.

6. Sardi JE, Giaroli A, Sananes C, et al. Long-term follow-up of the first randomized trial using neoadjuvant chemotherapy in stage $\mathrm{Ib}$ squamous carcinoma of the cervix:the final results. Gynecol Oncol. 1997;67(1):6169.

7. Walker AR, Walker BF, Siwedi D, et al. Low survival of South African urban black women with cervical cancer. $\mathrm{Br} J$ Obstet Gynaecol. 1985;92(12):1272-1278.

8. Wabinga H, Ramanakumar AV, Banura C, et al. Survival of cervical cancer patients in Kampala, Uganda:1995-1997. Br J Cancer. 2003;89(1):65-69.

9. Shepperd JH. Staging announcement:FIGO staging of gynecologic cancers;cervical and vulva. Int J Gynecol Cancer. 1995;5:319-325.

10. National Cancer Institute. Cancer Therapy Evaluation Program:Common toxicity criteria, version 3.0. Bethesda, Maryland, USA; 2003.

11. Kaplan EL, Meier P. Nonparametric estimation from incomplete observations. Journal of the American Statistical Association. 1958;53(282):457-481.

12. Freedman LS, Edwards BK, Ries LAG, et al. Cancer Incidence in Four Member Countries (MECC) compared with US SEER. National Cancer Institute. 2006;6:5873.

13. Stewart BW, Kleihues P. World Cancer Report. International Agency for Research Cancer. 2003.

14. Wabinga H, Ramana kumar AV, Banura C, et al. Survival of cervical cancer patients in Kampala, Uganda:1995-1997. Br J Cancer. 2003;89(1):65-69.

15. Ngwalle EW, Mgaya HN, Mpanju-Shumbusho W, et al. Situational analysis for diagnosis and treatment of cervical cancer in mainland Tanzania. East Afr Med J. 2001;78(2):60-64.

16. Herbert A, Singh N, Smith JA. Adenocarcinoma of the uterine cervix compared with squamous cell carcinoma: a 12-year study in Southampton and South-west Hampshire. Cytopathology. 2001;12(1):26-36.

17. American Cancer Society. Cancer facts and figures. American Cancer Society, Atlanta, USA;1999.

18. Keys HM, Bundy BN, Stehman FB, et al. Cisplatin, radiation, and adjuvant hysterectomy compared with radiation and adjuvant hysterectomy for bulky stage IB cervical carcinoma. $N$ Engl $J$ Med. 1999;340(15):1154-1161.

19. Whitney CW, Sause W, Bundy BN, et al. Randomized comparison of fluorouracil plus cisplatin versus hydroxyurea as an adjunct to radiation therapy in stage IIB-IVA carcinoma of the cervix with negative para aortic lymph nodes: a Gynecologic Oncology Group and Southwest Oncology Group study. J Clin Oncol. 1999;17(5):1339-1348.
20. Thomas GM. Concurrent chemotherapy and radiation for locally advanced cervical cancer:the new standard of care. Semin Radiat Oncol. 2000;10(1):44-50

21. Rose PG. Concurrent chemoradiation for locally advanced carcinoma of the cervix: where are we in 2006? Ann Oncol. 2006;17 (Suppl 10):x224 $\mathrm{x} 229$.

22. Eifel PJ, Winter K, Morris M, et al. Pelvic irradiation with concurrent chemotherapy versus pelvic and para-aortic irradiation for high-risk cervical cancer:an update of radiation therapy oncology group trial (RTOG) 90-01. J Clin Oncol. 2004;22(5):872-880.

23. Perez CA, Grigsby PW, Camel HM, et al. Irradiation alone or combined with surgery in stage IB, IIA and IIB carcinoma of uterine cervix:Update of a non randomised comparison. Int J Radiat Oncol Biol Phys. 1995;31(4):703-716.

24. Kim RY, Trotti A, Wu CJ Soong SJ, et al. Radiation alone in the treatment of cancer of the uterine cervix;Analysis of pelvic failure and dose response relationship. Int J Radiat Oncol Biol Phys. 1989;17(5):973978

25. Volterrani F, Feltre L, Sigurta D, et al . Radiotherapy versus surgery in the treatment of cervix stage Ib cancer. Int J Radiat Oncol Biol Phys. 1983;9(12):1781-1784.

26. Landoni F, Maneo A, Colombo A, et al. Randomised study of radical surgery versus radiotherapy for stage Ib-IIa cervical cancer. Lancet. 1997;350(9077):535-540.

27. Ayhan A, Tuncer ZS. Radical hysterectomy with lymphadenectomy for treatment of early stage cervical cancer:clinical experience of 278 cases. J Surg Oncol. 1991;47(3):175-177.

28. Montana GS, Fowler WC, Varia MA, et al. Carcinoma of the Cervix, Stage III Results of Radiation Therapy. Cancer. 1986;57(1):148-154.

29. Mascarello KC, Zandonade E, Amorim MH. Survival analysis of women with cervical cancer treated at a referral hospital for oncology in Espírito Santo State, Brazil, 2000-2005. Cad Saude Publica. 2013;29(4):823831.

30. Eifel PJ, Burke TW, Morris M, Smith TL. Adenocarcinoma as an independent risk factor for disease recurrence in patients with stage IB cervical carcinoma. Gynecol Oncol. 1995;59(1):38-44.

31. Sevin BU, Nadji M, Lampe B, et al. Prognostic factors of early stage cervical cancer treated by radical hysterectomy. Cancer. 1995;76(10 Suppl):1978-1986.

32. Aoki Y, Sasaki M, Watanabe M, et al. High-risk group in node-positive patients with stage IB, IIA, and IIB cervical carcinoma after radical hysterectomy and postoperative pelvic irradiation. Gynecol Oncol. 2000;77(2):305-309.

33. Trattner M, Graf AH, Lax S, et al. Prognostic factors in surgically treated stage ib-iib cervical carcinomas with special emphasis on the importance of tumor volume. Gynecol Oncol. 2001;82(1):11-16.

34. Kristensen GB, Abeler VM, Risberg B,. Tumor size, depth of invasion, and grading of the invasive tumor front are the main prognostic factors in early squamous cell carcinoma. Gynecol Oncol. 1999;74(2):245-251.

35. Ayhan A, Al RA, Baykal C, et al. A comparison of prognoses of FIGO stage IB adenocarcinoma and squamous cell carcinoma. Int J Gynecol Cancer. 2004;14(2):279-285. 